\title{
Abiotic stress and tissue-specific reference genes for quantitative reverse transcription PCR analysis in Korean native watermelons, Citrullus lanatus 'Black-King' and 'Speed-Plus-Honey'
}

\author{
Soo Jin Kim ${ }^{1,2} \cdot$ Il Lae Jung ${ }^{2} \cdot$ Hye-Eun Lee ${ }^{3}$. \\ Ji-Hoon Lee ${ }^{1}$ (D)
}

Received: 7 June 2018/Accepted: 6 August 2018/Published online: 17 August 2018

(C) The Korean Society for Applied Biological Chemistry 2018

\begin{abstract}
A wide variety of research on watermelon has been conducted, and such studies have been motivated by the published genome sequence database of watermelon. Screening of proper reference genes is the primary step for normalization in gene expression analysis. Based on previous studies conducted on Arabidopsis and cucumber, we selected eight candidate reference genes of $C l A C T, C l E F 1 \alpha$, ClGAPDH, ClIDH, ClLUG, ClPTB, ClUBC2, and Cl18SrRNA, respectively, encoding $\beta$-Actin, elongation factor 1- $\alpha$, glyceraldehyde-3-phosphate-dehydrogenase, NADP-isocitrate dehydrogenase, leunig, polypyrimidine tract-binding protein1, ubiquitin-conjugating enzyme E2, and $18 \mathrm{~S}$ ribosomal RNA from watermelon (Citrullus lanatus). The expression levels of these eight genes were evaluated by RT-qPCR under plant hormone-treatment $(100 \mu \mathrm{M}$ ABA) and abiotic stresses such as drought, cold $\left(4{ }^{\circ} \mathrm{C}\right)$, and high salt concentration $(250 \mathrm{mM} \mathrm{NaCl})$. The expression patterns of these eight genes were further compared across different types of watermelon tissues such as flower, leaf,
\end{abstract}

Electronic supplementary material The online version of this article (https://doi.org/10.1007/s13765-018-0391-3) contains supplementary material, which is available to authorized users.

Ji-Hoon Lee

jhlee2@jbnu.ac.kr

1 Departments of Bioenvironmental Chemistry, Chonbuk National University, Jeonju 54896, Republic of Korea

2 Department of Radiation Biology, Environmental Radiation Research Group, Korea Atomic Energy Research Institute, Daejeon 34057, Republic of Korea

3 Vegetable Crop Research Division, Horticultural Department, National Institute of Horticultural and Herbal Science (NIHHS), Rural Development Administration (RDA), 100, Nongsaengmyeong-ro, Iseo-myeon, Wanju-gun, Jeollabuk-do, Republic of Korea tendril, stem, root, and whole seedling. Our results showed that expressions of ClACT and ClEF1 $\alpha$, respectively in the Korean native watermelon cultivars Citrullus lanatus 'Black-King' and 'Speed-Plus-Honey' were least affected by the environmental stresses regardless of tissue types. Here, we suggest two ideal reference genes for watermelon RT-qPCR-based gene expression study.

Keywords Loading control gene - Reference gene $\cdot$ RTqPCR · Watermelon

\section{Introduction}

Watermelon (Citrullus lanatus) is one of the most consumed horticultural cucurbitaceous crops and occupies the third place after cucumber and melon, of which the whole genome sequence has been released. Genome sequence and transcriptome data would provide much better opportunities than before for conducting molecular biological studies on crops including watermelon (http://cucurbitgenomics.org) [1, 2].

Quantitative reverse transcription PCR (RT-qPCR) has become one of the standard experimental methods for studying gene transcription owing to its accuracy and specificity [3]. In molecular biology, gene expression analysis is broadly performed for multiple experimental purposes including identification of novel gene functions. The results of RT-qPCR depend on transcript normalization of the selected reference genes, which allow regulation of potential variations, caused by exposure to the same conditions as target genes. Therefore, chosen reference genes should be inspected for minimal variability in relative gene expression levels, compared to the experimental samples in advance to the RT-qPCR analysis [4]. 
Several suitable reference genes have been identified for some plants including Arabidopsis [5]. However, there is no universal reference gene, because the transcription levels of reference genes vary among tissues under different experimental conditions [6]. Moreover, the use of inappropriate reference gene influences the analysis and interpretation of transcription patterns of target genes, resulting in misunderstanding of the functions of the target genes $[7,8]$. Therefore, selection of appropriate reference genes is essential for producing correct and reliable results in RT-qPCR experiment.

Stable reference genes in watermelon have not been reported with respect to specific experimental conditions such as drought, cold, and salt stresses. In this research, eight candidate reference genes previously described in watermelons, including of ClACT, ClEFl $\alpha, C l G A P D H$, ClIDH, ClLUG, ClPTB, ClUBC2, and Cll8SrRNA, respectively, encoding $\beta$-Actin, elongation factor $1-\alpha$, glyceraldehyde-3-phosphate-dehydrogenase, NADP-isocitrate dehydrogenase, leunig, polypyrimidine tract-binding protein1, ubiquitin-conjugating enzyme $\mathrm{E} 2$, and $18 \mathrm{~S}$ ribosomal RNA were chosen, and their transcripts were quantified by RT-qPCR in watermelon tissues under a broad spectrum of stress conditions [9].

\section{Materials and methods}

\section{Plant materials}

Seeds of watermelon cultivars Citrullus lanatus. 'BlackKing' and 'Speed-Plus-Honey' were obtained from the Nongwoobio Seed Company (Suwon, Republic of Korea). To study the effects of abiotic stresses on seedling growth, Black-King (BK) and Speed-Plus-Honey (SPH) seeds (commercial F1 hybrid) were sterilized with $30 \%$ sodium hypochlorite (bleach) for 15-20 min, rinsed for 10 times with distilled water, germinated in Murashige and Skoog (MS) medium with $3 \%$ sucrose $(\mathrm{pH} 5.8$ ) and $0.8 \%$ phytoagar, and grown in a growth chamber at $25{ }^{\circ} \mathrm{C}$ under long-day conditions ( $16 \mathrm{~h}$ light and $8 \mathrm{~h}$ dark) [10]. The watermelon plants used for the tissue-specific expression pattern analysis were cultured in a greenhouse [11].

\section{Abiotic stress treatment and sampling}

The BK and SPH watermelon seedlings were subjected to drought, cold, salt, and abscisic acid (ABA) treatments. The BK and SPH watermelons were raised in agar plates for 2 weeks. The harvested whole seedlings from the plates were dehydrated on 3MM filter paper (Whatman) at around $25{ }^{\circ} \mathrm{C}$ and approximately $60 \%$ humidity under dim light. The 2-week-old seedlings were soaked in the MS liquid media containing $300 \mathrm{mM} \mathrm{NaCl}$ for salt stress, or $100 \mu \mathrm{M}$ $\mathrm{ABA}$ for $\mathrm{ABA}$ treatment for $2 \mathrm{~h}$. For cold stress treatment, the watermelon seedlings were transferred to an incubator set at $4{ }^{\circ} \mathrm{C}$ for $6 \mathrm{~h}$. Whole seedlings were harvested, promptly soaked in liquid $\mathrm{N}_{2}$, and stored at $-80{ }^{\circ} \mathrm{C}$ until extraction of the total RNA from samples $[12,13]$.

\section{Selection of candidate reference genes and primer design for $\mathrm{qPCR}$}

To identify proper loading control genes for watermelon, eight candidate genes were evaluated. The eight genes were chosen based on previous studies conducted on watermelon and other crops. The selected genes were ClACT, ClEFI $\alpha, C l G A P D H, C l I D H, C l L U G, C l P T B$, $C l U B C 2$, and Cll8SrRNA as described earlier. To obtain orthologous reference genes in watermelon, Arabidopsis homologs of the selected eight genes were used for blastn search against watermelon genomes in the Cucurbit Genomics Database. The coding DNA sequences with the best hit were chosen and uploaded to Beacon Designer (http://www.premierbiosoft.com/molecular_beacons/) and Primer3Plus software (http://primer3plus.com) for designing primers. The primers of the loading control genes used for RT-qPCR in this study were designed following previously described protocols [9]. Information on the chosen reference genes is listed in Table 1. For more comparable results, the primer pair for $18 \mathrm{~S}$ rRNA gene, which was previously published, was used in this study [1]. Results on the selected eight genes for loading control and their amplification characters are listed in Tables 1 and 2.

\section{Total RNA extraction, RT-PCR, and RT-qPCR}

Total RNA of the watermelon was extracted from drought-, cold-, and salt-stressed and ABA-treated 14-day-old watermelon seedlings using a commercial RNA extraction kit (Intron Biotech., Daejeon, Republic of Korea) according to the protocol. cDNA synthesis and RT-PCR were performed following previously described protocols [14]. Real-time RT-qPCR was performed on a CFX96 Real-time System (Bio-Rad, Hercules, CA, USA), and data of RTqPCR were analyzed with CFX Manager program (BioRad). PCR reactions were conducted as described previously [13]. The PCR cycling conditions were described previously [12]. The melting curves were recorded after forty cycles to test primer specificity by heating from 55 to $95{ }^{\circ} \mathrm{C}$. Three replicates were conducted for each sample. Negative controls without any cDNA as templates were included. Amplification efficiencies of RT-qPCR for all primer pairs for eight candidates genes were evaluated using the serial tenfold dilutions of the pooled cDNAs $(0.5$, 5,50 , and $500 \mathrm{ng}$ ) [15]. 
Table 1 Description of watermelon candidate reference genes for qRT-PCR

\begin{tabular}{lllll}
\hline Genes & Descriptions & IDs & Homologue locus for Arabidopsis & E-value \\
\hline ClACT & $\beta$-actin & Cla007792 & At5g09810 & 0 \\
ClEF1 $\alpha$ & Elongation factor 1- $\alpha$ & Cla010539 & At1g07940 & 0 \\
$C l G A P D H$ & Glyceraldehyde-3-phosphate-dehydrogenase & Cla013454 & At1g16300 & $1 \mathrm{e}-105$ \\
$C l I D H$ & NADP-isocitrate dehydrogenase & Cla009135 & At1g65930 & $1 \mathrm{e}-71$ \\
$C l L U G$ & Leunig & Cla022288 & At4g32551 & $7 \mathrm{e}-62$ \\
$C l P T B$ & Polypyrimidine tract-binding protein1 & Cla004906 & At5g53180 & $5 \mathrm{e}-92$ \\
$C l U B C 2$ & Ubiquitin-conjugating enzyme E2 & Cla010164 & At2g02760 & $4 \mathrm{e}-81$ \\
$C l 18 S R R N A$ & 18S ribosomal RNA & & & \\
\hline
\end{tabular}

Table 2 Information about primer sets of watermelon candidate reference genes for qRT-PCR

\begin{tabular}{|c|c|c|c|c|}
\hline$\overline{\text { Genes }}$ & Primer sequences $5^{\prime}-3^{\prime}$ & Product size (bp) & Amplification efficiency (\%) & $R^{2}$ \\
\hline \multirow[t]{2}{*}{ ClACT } & F:CCATGTATGTTGCCATCCAG & 140 & 93.4 & 1.000 \\
\hline & R:GGATAGCATGGGGTAGAGCA & & & \\
\hline \multirow[t]{2}{*}{ ClEF1a } & F:GCACGCTCTTCTTGCTTTC & 115 & 89.6 & 1.000 \\
\hline & R:ACGATTTCGTCGTACCTTGC & & & \\
\hline \multirow[t]{2}{*}{$C l G A P D H$} & F:CTGGCAGTACTTTGCCAACA & 87 & 97.5 & 0.999 \\
\hline & R:AGGATTGGAGAGGAGGTCGT & & & \\
\hline \multirow[t]{2}{*}{ ClIDH } & F:TGGCCTCTTTACCTAAGCACA & 124 & 92.1 & 1.000 \\
\hline & R:ATATGCCAGCAGCCTCAAAC & & & \\
\hline \multirow[t]{2}{*}{ ClLUG } & F:TTGCTGGTCATTGGATGCTA & 138 & 86.8 & 0.995 \\
\hline & R:GCCGAAGCAACTAGACCTGA & & & \\
\hline \multirow[t]{2}{*}{ ClPTB } & F:GGAGCAAACAGAAATCAAGC & 133 & 93.5 & 0.997 \\
\hline & R:AGCAGGCTCAGAGGAAGATG & & & \\
\hline \multirow[t]{2}{*}{$C l U B C 2$} & F:CCAAATAAGCCACCGACAGT & 118 & 95 & 0.999 \\
\hline & R:TCATAGATTGGGCTCCATTG & & & \\
\hline \multirow[t]{2}{*}{ Cl18SrRNA } & F:AGCCTGAGAAACGGCTACCACATC & & 52.6 & 0.999 \\
\hline & R:ACCAGACTCGAAGAGCCCGGTAT & & & \\
\hline
\end{tabular}

\section{Results and discussion}

\section{Conditions for PCR amplification of the reference genes}

From the watermelon genome sequence, homologous genes were obtained by examining sequence similarity hits. All the primers' information used in this study is listed in Tables 1 and 2.

Results of melting curve analysis are shown in Fig. S1 (in Supplemental Material). The presence of a single peak without primer-dimer formation indicates only amplification specificity for each reference gene.

Standard curve result analysis determined the efficiency of the real-time RT-qPCR for each primer pair and was generated by using four serial tenfold dilutions of cDNA, ranging from $52.6 \%$ (Cl18SrRNA) to $97.5 \%$ (ClGAPDH).
The determination of coefficients $\left(R^{2}\right)$ of the standard curve regression equation varied from $0.995(C l L U G)$ to 1.000 (ClACT, ClEF1 $\alpha$, and ClIDH) (Table 2). The results implied that specific and efficient RT-qPCR systems were established to quantify the selected reference genes, except Cl18srRNA.

\section{Gene expression analysis}

The mRNA levels of eight reference genes in two cultivars, Citrullus lanatus 'Black-King (BK)' and 'Speed-Plus Honey (SPH)' expressed in watermelon seedlings subjected to a broad spectrum of abiotic (drought, cold, and salinity) and ABA hormone stresses, are presented as quantification cycle $\left(C_{\mathrm{q}}\right)$ values in Fig. S2. Different transcript abundances were observed for the eight genes. However, the mRNA transcription levels of the genes in 

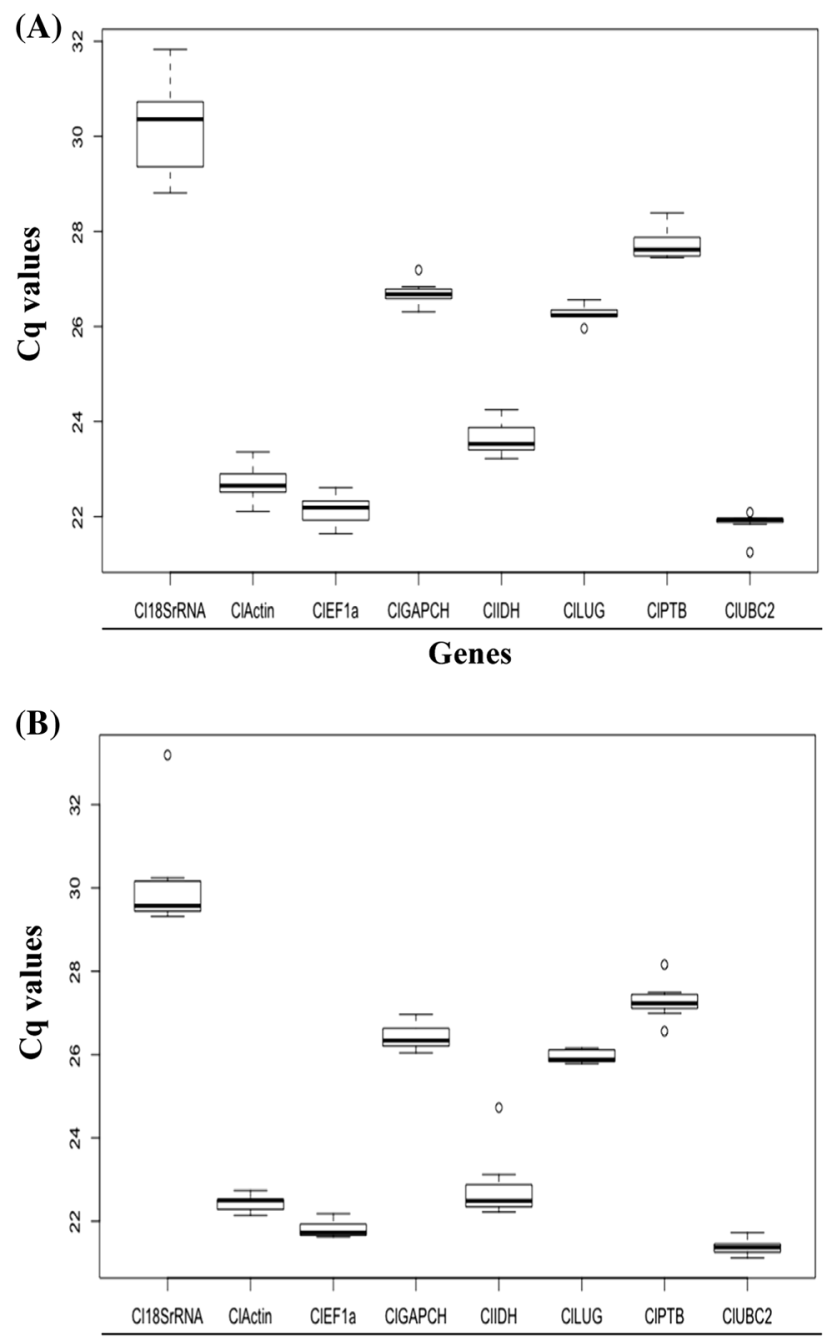

Genes

Fig. 1 Expression profiles of watermelon candidate reference genes. The mean $C_{\mathrm{q}}$ values of all the stress experiments in each gene are presented with RSD values for Black-King (A) and Speed-Plus-

the two varieties, BK and SPH, were almost the same. Cl18SrRNA, which had the highest mean $C_{\mathrm{q}}$ value of 30 for both the BK and SPH varieties, had the lowest mRNA expression level among all the candidate genes. The expression levels for ClACT, ClEF $1 \alpha, C l I D H$, and $C l U B C 2$ were similar and ranged from 21 to 22 cycles. The expression levels for $C l G A P D H, C l L U G$, and $C l P T B$ were comparable and ranged from 25 to 27 cycles. The average $C_{\mathrm{q}}$ values of $C l A C T, C l E F 1 \alpha$, and $C l U B C 2$ were within the range of 21 to 22 cycles. Moreover, these three genes, among the eight selected loading control genes, exhibited a constant expression level in all the stressed seedlings. The variability of $C_{\mathrm{q}}$ values in the abiotic stress-treated samples was highest for ClI8SrRNA and ClIDH, whereas ClACT, $C l E F 1 \alpha$, and $C l U B C 2$ showed the lowest variations in gene expression (Fig. S2).

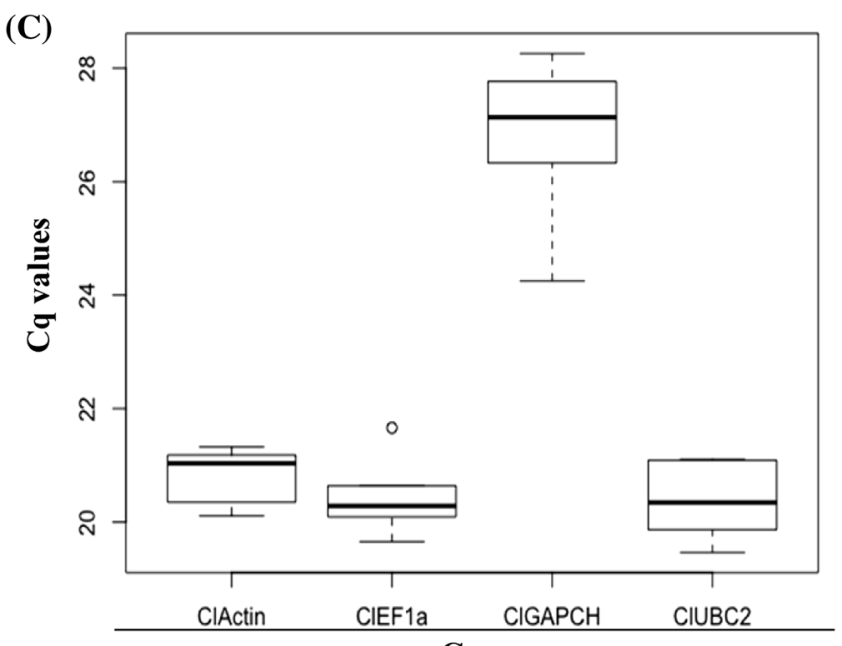

Genes

(D)

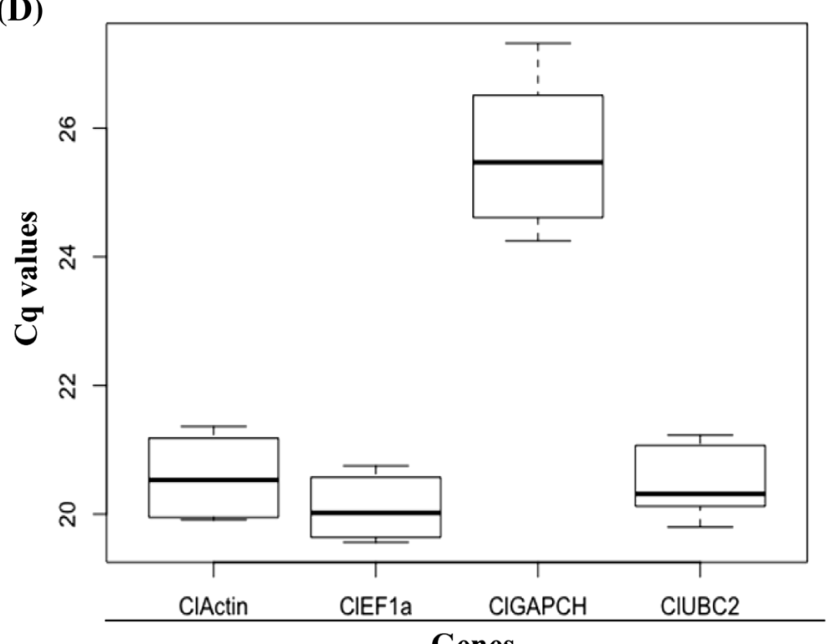

Genes

Honey (B). The average $C_{\mathrm{q}}$ values per tissue in each gene are also presented with RSD values for Black-King (C) and Speed-PlusHoney (D)

To get reliable reference genes, the $C_{\mathrm{q}}$ values of those eight selected reference genes in the abiotic stress-treated samples were evaluated by the standard deviation (SD) and relative standard deviation (RSD) values. In Fig. 1, the RSD values of the eight genes were from 0.93 to $4.54 \%$, which were all in the acceptable range [16]. Especially, the RSD values of $C l A C T, C l E F 1 \alpha$, and $C l U B C 2$ were less than $1 \%$. Therefore, the ClACT, ClEF1 $\alpha$, and $C l U B C 2$ genes were selected for further analysis.

To elucidate the variability of transcript levels of $C l A C T, C l E F 1 \alpha, C l U B C 2$, and $C l G A P D H$ in the flowers, leaves, tendrils, stems, roots, and whole seedlings of watermelons SPH and BK, RT-qPCR analysis was carried out (Fig. S3). Average $C_{\mathrm{q}}$ values of $C l A C T, C l E F 1 \alpha$, or $C l U B C 2$ among the different tissues were comparable to each other with minor variations. Average $C_{\mathrm{q}}$ values of $C l G A P D H$ ranged from 24 to 28 in tissues of two different 
watermelon cultivars which were used in this paper. Therefore, this gene was not considered proper as a tissuespecific reference gene, because the difference of $C_{\mathrm{q}}$ was about 3 to 4 . As a result, ClACT, ClEF $1 \alpha$, and ClUBC2 were considered appropriate reference genes for tissuespecific study.

In case of BK watermelon, ClACT would be the most proper reference gene when tissue-specific gene expression test would be performed. The gene ClEFl $\alpha$ was the most appropriate as the reference gene in tissue-specific transcription level test of SPH watermelon. These two genes were selected on the basis of the least SD and RSD values. The SD and RSD values of BK watermelon were $0.49 \%$ and $2.35 \%$, respectively. The SD and RSD values of SPH watermelon were $0.60 \%$ and $2.93 \%$, respectively (Fig. 1). Therefore, we suggest ClACT of BK and ClEF $1 \alpha$ of SPH as reference genes for the tissue-specific gene expression experiment. Considering the $C_{\mathrm{q}}, \mathrm{SD}$, and RSD values of the diverse abiotic stresses and tissues, it seems that ClACT gene is suitable as a reference gene for BK watermelon and ClEF1 $\alpha$ gene for SPH watermelon.

In cucumbers, one of the cucurbitaceous crops, the genes of EFI $\alpha, F$-box, and CAC (Clathrin Adaptor Complex Subunit) have been reported to be stable genes under diverse abiotic stresses and growth regulatory treatments [17]. In another study on cucumbers, the genes $E F 1 \alpha, U B I$ ep (Ubiquitin like protein), and TUA ( $\alpha$-Tubulin) were also shown to be most stable [18]. For melons, genes encoding ribosomal protein L2 (RPL2), actin ( $A C T)$, and cyclophilin (Cyp) were the three best loading control genes, when melon stems were infected with Fusarium wilt [19].

In conclusion, among the eight watermelon genes studied here, ClACT and ClEFl $\alpha$ were found to be suitable reference genes, respectively, for $\mathrm{BK}$ and $\mathrm{SPH}$ watermelons in order to study normalization of gene expression in watermelon under many kinds of environmental stresses and diverse tissues. The optimal reference genes selected in watermelon were different from the reference genes in cucumber and melon, even though these three are all cucurbitaceous crops. Moreover, watermelon had different reference genes that were suitable for different varieties. Therefore, we suggest that $C l A C T$ and $C l E F 1 \alpha$ genes would be suitable as the reference genes for BK and SPH watermelons, respectively.

Acknowledgments This research was supported by grants from the Radiation Technology R\&D program (Project No. NRF2015M2A2B2034284) through the National Research Foundation of Korea funded by the Ministry of Science, ICT \& Future. This research was also supported by the National Institute of Horticultural \& Herbal Science, Rural Development Administration (RDA), Republic of Korea (Project No. PJ00926102).

\section{References}

1. Guo S, Liu J, Zheng Y, Huang M, Zhang H, Gong G, He H, Ren Y, Zhong S, Fei Z, Xu Y (2011) Characterization of transcriptome dynamics during watermelon fruit development: sequencing, assembly, annotation and gene expression profiles. BMC Genom 12:454

2. Guo S, Zhang J, Sun H, Salse J, Lucas WJ, Zhang H, Zheng Y, Mao L, Ren Y, Wang Z, Min J (2013) The draft genome of 21 watermelon (Citrullus lanatus) and resequencing of diverse accessions. Nat Genet 45:51-58

3. Gachon C, Mingam A, Charrier B (2004) Real-time PCR: what relevance to plant studies? J Exp Bot 55:1445-1454

4. Guénin S, Mauriat M, Pelloux J, Van Wuytswinkel O, Bellini C, Gutierrez L (2009) Normalization of qRT-PCR data: the necessity of adopting a systematic, experimental conditions-specific, validation of references. J Exp Bot 60:487-493

5. Czechowski T, Stitt M, Altmann T, Udvardi MK, Scheible W-R (2005) Genome-wide identification and testing of superior reference genes for transcript normalization in Arabidopsis. Plant Physiol 139:5-17

6. Hruz T, Wyss M, Docquier M, Pfaffl MW, Masanetz S, Lorenzo Borghi L, Verbrugghe P, Kalaydjieva L, leuler S, Laule O, Descombes P, Gruissem W, Zimmermann P (2011) RefGenes: identification of reliable and condition specific reference genes for RT-qPCR data normalization. BMC Genom 12:156

7. Ferguson BS, Nam H, Hopkins RG, Morrison RF (2010) Impact of reference gene selection for target gene normalization on experimental outcome using real-time qRT-PCR in adipocytes. PLoS One 5:e15208

8. Gutierrez L, Mauriat M, Guénin S, Pelloux J, Lefebvre JF, Louvet R, Rusterucci C, Moritz T, Guerineau F, Bellini C, Van Wuytswinkel O (2008) The lack of a systematic validation of reference genes: a serious pitfall undervalued in reverse transcription polymerase chain reaction (RT-PCR) analysis in plants. Plant Biotechnol J 6:609-618

9. Kim SJ, Huh YC, Ahn YK, Kim JH, Kim DS, Lee HE (2015) Watermelon (Citrullus lanatus) late-embryogenesis abundant group 3 protein, ClLEA3-1, responds to diverse abiotic stresses. Hortic Environ Biotechnol 56(4):555-560

10. Kong Q, Yuan J, Niu P, Xie J, Jiang W, Huang Y, Bie Z (2014) Screening suitable reference genes for normalization in reverse transcription quantitative real-time PCR analysis in Melon. PLoS One 9:e87197

11. Chung E, Kim SY, Yi SY, Choi D (2003) Capsicum annum dehydrin, an osmotic stress gene in hot pepper plants. Mol Cells 15:327-332

12. Kim SJ, Kim WT (2013) Suppression of Arabidopsis RING E3 ubiquitin ligase AtATL78 increases tolerance to cold stress and decreases tolerance to drought stress. FEBS Lett 587:2584-2590

13. Forghani AH, Almodares A, Ehsanpour AA (2018) Potential objectives for gibberellic acid and paclobutrazol under salt stress in sweet sorghum (Sorghum bicolor [L.] Moench cv. Sofra). Appl Biol Chem 61:113-124

14. Joo JS, Oh NL, Nguyen NH, Lee YH, Kim YK, Song SI, Cheong JJ (2017) Intergenic transformation of AtMYB44 confers drought stress tolerance in rice seedlings. Appl Biol Chem 60:447-455

15. Kim JH, Hong JY, Moon JC, Kwon KS, Jang CS (2018) Development of molecular markers for detecting almond, peanut, pine nut, and walnut in commercial food using quantitative realtime PCR. Appl Biol Chem 61:345-354

16. Li X, Wang X, Yang J, Liu Y, He Y, Pan L (2014) A novel quadruplex real-time PCR method for simultaneous detection of Cry2Ae and two genetically modified cotton events (GHB119 and T304-40). BMC Biotechnol 14:43 
17. Migocka M, Papierniak A (2011) Identification of suitable reference genes for studying gene expression in cucumber plants subjected to abiotic stress and growth regulators. Mol Breeding 28:343-357

18. Wan HJ, Zhao ZG, Qian CT, Sui YH, Malik AA (2010) Selection of appropriate reference genes for gene expression studies by quantitative real-time polymerase chain reaction in cucumber. Anal Biochem 399:257-261

19. Sestili S, Sebastiani MS, Belisario A, Ficcadenti N (2014) Reference gene selection for gene expression analysis in melon infected by Fusarium oxysporum f.sp. melonis. J Plant Biochem Biotechnol 23:238-248 